\title{
Neutral Slant Submanifolds of a Para-Kähler Manifold
}

\author{
Yılmaz Gündüzalp \\ Department of Mathematics, Dicle University, 21280 Diyarbakır, Turkey \\ Correspondence should be addressed to Yılmaz Gündüzalp; zygunduzalp@gmail.com
}

Received 27 May 2013; Accepted 9 August 2013

Academic Editor: Jaume Giné

Copyright (C) 2013 Yllmaz Gündüzalp. This is an open access article distributed under the Creative Commons Attribution License, which permits unrestricted use, distribution, and reproduction in any medium, provided the original work is properly cited.

We define and study both neutral slant and semineutral slant submanifolds of an almost para-Hermitian manifold and, in particular, of a para-Kähler manifold. We give characterization theorems for neutral slant and semi-neutral slant submanifolds. We also investigate the integrability conditions for the distributions involved in the definition of a semi-neutral slant submanifold when the ambient manifold is a para-Kähler manifold.

\section{Introduction}

The geometry of slant submanifolds was initiated by Chen, as a generalization of both holomorphic and totally real submanifolds in complex geometry $[1,2]$. Since then, many mathematicians have studied these submanifolds. Slant submanifolds have been studied by many geometers in various manifolds [3-5]. In particular, Papaghiuc [6] introduced semislant submanifolds. Lotta $[7,8]$ defined and studied slant submanifolds in contact geometry. Cabrerizo et al. studied slant, semislant, and bislant submanifolds in contact geometry $[9,10]$. Recently, Arslan et al. [11] studied these submanifolds in the setting of neutral Kähler manifolds.

In this paper we define and study both neutral slant and semineutral slant submanifolds of an almost para-Hermitian manifold and, in particular, of a para-Kähler manifold. The paper is organized as follows. In Section 2, we review some formulas and definitions for an almost para-Hermitian manifold and their submanifolds. In Section 3, we define neutral slant submanifolds for an almost para-Hermitian manifold and give theorem for a neutral slant submanifold. In the last section, we define and study semineutral slant submanifolds of an almost para-Hermitian manifold. We give theorems for a semineutral slant submanifold. In the last part of Section 4, we obtain that the distributions are integrable and their leaves are totally geodesic in semineutral slant submanifold under the condition $\nabla f=0$. Finally, the paper contains some examples.

\section{Preliminaries}

An almost para-Hermitian manifold $(\bar{M}, g, J)$ is a smooth manifold endowed with an almost paracomplex structure $J$ and a pseudo-Riemannian metric $g$ compatible in the sense that

$$
J^{2}=I, \quad g(J X, Y)+g(X, J Y)=0, \quad X, Y \in \Gamma(T \bar{M}),
$$

where $\Gamma(T M)$ is the module of differentiable vector fields on $M$. It follows that the metric $g$ is neutral; that is, it has signature $(m, m)$, and the eigenbundles $T \bar{M}^{ \pm}$are totally isotropic with respect to $g$.

An almost para-Hermitian manifold $\bar{M}$ is called a paraKähler manifold if

$$
\left(\bar{\nabla}_{X} J\right) Y=0, \quad \forall X, Y \in \Gamma(T \bar{M}),
$$

where $\bar{\nabla}$ is the Levi-Civita connection on $\bar{M}[12,13]$.

Let $M$ be an isometrically immersed submanifold of an almost para-Hermitian manifold $\bar{M}$. We denote the LeviCivita connections on $M$ and $\bar{M}$ by $\nabla$ and $\bar{\nabla}$, respectively. Then, the Gauss and Weingarten formulas are given by

$$
\begin{aligned}
& \bar{\nabla}_{X} Y=\nabla_{X} Y+h(X, Y), \\
& \bar{\nabla}_{X} N=-A_{N} X+\nabla_{X}^{\perp} N,
\end{aligned}
$$


for any $X, Y \in \Gamma(T M)$ and $N \in \Gamma\left(T M^{\perp}\right)$, where $\nabla^{\perp}$ is the connection in the normal bundle $T M^{\perp}, h$ is the second fundamental form of $M$, and $A_{N}$ is the shape operator. The second fundamental form $h$ and the shape operator $A_{N}$ are related by

$$
g\left(A_{N} X, Y\right)=g(h(X, Y), N),
$$

where the induced pseudo-Riemannian metric on $M$ is denoted by the same symbol $g$.

Let us consider that $M$ is an immersed submanifold of an almost para-Hermitian manifold $\bar{M}$. For any $X \in \Gamma(T M)$ and $N \in \Gamma\left(T M^{\perp}\right)$, we put

$$
\begin{aligned}
& J X=f X+\omega X, \\
& J N=B N+C N,
\end{aligned}
$$

where $f X$ (resp., $\omega X$ ) is tangential (resp., normal) part of $J X$ and $B N$ (resp., $C N$ ) is tangential (resp., normal) part of $J N$. From (1) and (5), we have

$$
g(f X, Y)=-g(X, f Y),
$$

for any $X, Y \in \Gamma(T M)$.

The submanifold $M$ is said to be invariant if $\omega$ is identically zero; that is, $J X=f X \in \Gamma(T M)$, for any $X \in$ $\Gamma(T M)$. On the other hand, $M$ is said to be anti-invariant submanifold if $f$ is identically zero; that is, $J X=\omega X \in$ $\Gamma\left(T M^{\perp}\right)$, for any $X \in \Gamma(T M)$.

For any $X \in \Gamma(T M)$, by a direct calculation, we have

$$
\begin{aligned}
& X=f^{2} X+B \omega X, \quad \text { that is, } I=f^{2}+B \omega, \\
& \omega f X+C \omega X=0, \quad \text { that is, } \omega f+C \omega=0 .
\end{aligned}
$$

Similarly, for any $N \in \Gamma\left(T M^{\perp}\right)$, we have

$$
\begin{aligned}
& N=\omega B N+C^{2} N, \quad \text { that is, } I=\omega B+C^{2}, \\
& f B N+B C N=0, \quad \text { that is, } f B+B C=0 .
\end{aligned}
$$

Now, let $M$ be an immersed submanifold of an almost para-Kähler manifold $\bar{M}$. For any $X, Y \in \Gamma(T M)$, from $\bar{\nabla}_{X} J Y=J\left(\bar{\nabla}_{X} Y\right)$, taking into account (3), (5), and (6), then we have

$$
\begin{aligned}
\nabla_{X} f Y & +h(X, f Y)-A_{\omega Y} X+\nabla_{X}^{\perp} \omega Y \\
& =f \nabla_{X} Y+\omega \nabla_{X} Y+B h(X, Y)+C h(X, Y) .
\end{aligned}
$$

Comparing the tangential and normal components of (11), respectively, we get

$$
\begin{gathered}
\left(\nabla_{X} f\right) Y=A_{\omega Y} X+B h(X, Y), \\
\left(\nabla_{X} \omega\right) Y=C h(X, Y)-h(X, f Y),
\end{gathered}
$$

where the covariant derivations of $f$ and $\omega$ are, respectively, defined by

$$
\begin{aligned}
& \left(\nabla_{X} f\right) Y=\nabla_{X} f Y-f \nabla_{X} Y, \\
& \left(\nabla_{X} \omega\right) Y=\nabla_{X}^{\perp} \omega Y-\omega \nabla_{X} Y,
\end{aligned}
$$

for any $X, Y \in \Gamma(T M)$.
Let $M$ be a submanifold of a para-Hermitian manifold $\bar{M}$. A tangent vector $X \in T M$ is said to be spacelike (resp., timelike) if $g(X, X)>0$ (resp., $g(X, X)<0$ ). If $X$ is a spacelike vector (resp., timelike), we have $\|X\|=\sqrt{g(X, X)}$ (resp., $\|X\|=\sqrt{-g(X, X)})[11]$.

\section{Neutral Slant Submanifolds of Almost Para-Hermitian Manifolds}

In this section, we study neutral slant immersions of an almost para-Hermitian manifold $\bar{M}$. First, we present definition of a neutral slant submanifold of an almost paraHermitian manifold following Chen's [1] definition for a Hermitian manifold. Let $M$ be a semi-Riemannian manifold isometrically immersed in an almost para-Hermitian manifold $\bar{M}$. For each nonzero spacelike vector $X$ tangent to $M$ at $x$, the angle $\theta(X), 0 \leq \theta(X) \leq \pi / 2$ between $J X$ and $T_{x} M$ is called the Wirtinger angle of $X$. Then, $M$ is said to be neutral slant if the angle $\theta(X)$ is a constant, which is independent of the choice of $x \in M$ and $X \in \Gamma(T M)$. The angle $\theta$ of a neutral slant immersion is called the slant angle of the immersion. Thus, the invariant and anti-invariant immersions are neutral slant immersions with slant angle $\theta=0$ and $\theta=\pi / 2$, respectively. A neutral slant immersion which is neither invariant nor anti-invariant is called a proper neutral slant immersion.

We note that our definition is quite different from Chen's definition for slant submanifold [1], and the slant submanifold is given by Arslan et al. [11].

Next we give a useful characterization of neutral slant submanifolds in an almost para-Hermitian manifold.

Theorem 1. Let $M$ be a submanifold of a para-Hermitian manifold $\bar{M}$. Then,

(i) $M$ is neutral slant if and only if there exists a constant $\lambda \in[0,1]$ such that $f^{2}=\lambda I$. Furthermore, in this case, if $\theta$ is the slant angle of $M$, it satisfies $\lambda=\cos ^{2} \theta$;

(ii) $M$ is a neutral slant submanifold if and only if there exists a constant $\lambda \in[0,1]$ such that $B^{2} \omega=\lambda I$. Furthermore, in this case, if $\theta$ is the slant angle of $M$, it satisfies $\lambda=\sin ^{2} \theta$.

Proof. (i) Suppose that $M$ is a neutral slant submanifold. For any $X \in \Gamma(T M)$, we can write

$$
\cos \theta(X)=\frac{\|f X\|}{\|J X\|}
$$

where $\theta(X)$ is the slant angle. By using (7), (15), and (1), we get

$$
\begin{aligned}
g\left(f^{2} X, X\right) & =-g(f X, f X) \\
& =-\cos ^{2} \theta(X) g(J X, J X) \\
& =\cos ^{2} \theta(X) g(X, X),
\end{aligned}
$$


for all $X \in \Gamma(T M)$. Since $g$ is a neutral metric, from (16), we have

$$
f^{2} X=\cos ^{2} \theta(X) X, \quad X \in \Gamma(T M) .
$$

Let $\lambda=\cos ^{2} \theta$. Then it is obvious that $\lambda \in[0,1]$.

Conversely, let us assume that there exists a constant $\lambda \epsilon$ $[0,1]$ such that $f^{2}=\lambda I$ is satisfied. From (7), (17), and (1), we get

$$
\begin{aligned}
\cos \theta(X) & =\frac{g(J X, f X)}{\|J X\|\|f X\|}=-\frac{g\left(X, f^{2} X\right)}{\|J X\|\|f X\|} \\
& =-\lambda \frac{g\left(X, J^{2} X\right)}{\|J X\|\|f X\|}=\lambda \frac{g(J X, J X)}{\|J X\|\|f X\|},
\end{aligned}
$$

for all $X \in \Gamma(T M)$. Thus we have

$$
\cos \theta(X)=\frac{\lambda\|J X\|}{\|f X\|} .
$$

Since $\cos \theta(X)=\|f X\| /\|J X\|$, then by using the last equation we obtain $\cos ^{2} \theta(X)=\lambda$, which implies that $\theta(X)$ is a constant and so $M$ is a neutral slant.

(ii) From (8) and (i), we have (ii).

Corollary 2. Let $M$ be a neutral slant submanifold of an almost para-Hermitian manifold $\bar{M}$ with slant angle $\theta$. Then, for any $X, Y \in \Gamma(T M)$, we have

$$
\begin{gathered}
g(f X, f Y)=-\cos ^{2} \theta g(X, Y), \\
g(\omega X, \omega Y)=-\sin ^{2} \theta g(X, Y) .
\end{gathered}
$$

Proof. From Theorem 1(i) and (7), we get

$$
\begin{gathered}
g(f X, f Y)=-g\left(f^{2} X, Y\right), \\
g(f X, f Y)=-\cos ^{2} \theta g(X, Y),
\end{gathered}
$$

for any $X, Y \in \Gamma(T M)$. On the other hand, from (1), (5), and (20), we obtain

$$
\begin{gathered}
g(J X, J Y)=g(f X+\omega X, f Y+\omega Y), \\
-g(X, Y)=g(f X, f Y)+g(\omega X, \omega Y) .
\end{gathered}
$$

This completes the proof.

Now, we give some examples of the neutral slant submanifolds in almost para-Hermitian manifolds inspirited by Chen [1].

Note that given a semi-Euclidean space $R_{n}^{2 n}$ with coordinates $\left(x_{1}, \ldots, x_{2 n}\right)$ on $R_{n}^{2 n}$, we can naturally choose an almost paracomplex structure $J$ on $R_{n}^{2 n}$ as follows:

$$
J\left(\frac{\partial}{\partial x_{2 i}}\right)=\frac{\partial}{\partial x_{2 i-1}}, \quad J\left(\frac{\partial}{\partial x_{2 i-1}}\right)=\frac{\partial}{\partial x_{2 i}},
$$

where $i=1, \ldots, n$. Let $R_{n}^{2 n}$ be a semi-Euclidean space of signature $(+,-,+,-, \ldots)$ with respect to the canonical basis $\left(\partial / \partial x_{1}, \ldots, \partial / \partial x_{2 n}\right)$.
Example 3. Consider a submanifold $M$ in $R_{2}^{4}$ given by

$$
\varphi(u, v)=(u \cos \alpha, v, u \sin \alpha, 0) .
$$

It is easy to see that $M$ is a neutral slant submanifold with the slant angle $\alpha$.

Example 4. Consider a submanifold $M$ in $R_{2}^{4}$ given by

$$
x(u, v)=(u \sin \alpha, v \cos \beta, u \cos \alpha, v \sin \beta),
$$

where $\alpha$ and $\beta$ are constant. Then $M$ is a neutral slant submanifold with the slant angle $\cos \theta=|\sin (\alpha+\beta)|$.

Remark 5. Consider $M_{p}^{2 p}$ a neutral submanifold of an almost para-Hermitian manifold $(\bar{M}, g, J)$, in fact a neutral manifold $\bar{M}_{n}^{2 n}$, with

$$
|g(f X, J X)| \leq\|f X\|\|J X\| \text {. }
$$

$M$ is called a neutral slant submanifold if the Wirtinger angle between $J X$ and $T_{x} M$ is constant, for all $X \in T_{x} M$ a spacelike vector field and all $x \in M$. It is well defined, because that angle can be measured as usual, it the same angle between $J X$ and $f X$, and they both are timelike vector fields.

In fact, if that conditions hold, it would be the same angle between $J Y$ and $T_{x} M$ for $Y \in T_{x} M$ a timelike vector, both $J Y$ and $f Y$ would be spacelike vector fields. This condition is equivalent to

$$
|g(f X, f X)| \leq|g(J X, J X)|,
$$

or $\|f X\| \leq\|J X\|$, in fact it is equivalent to Theorem 1 condition $f^{2} X=\cos ^{2} \theta I$.

\section{Semineutral Slant Submanifolds of Almost Para-Hermitian Manifolds}

Definition 6. Let $(\bar{M}, g)$ be an almost para-Hermitian manifold with an almost paracomplex structure $J$. A differentiable distribution on $\bar{M}$ is called a neutral slant distribution if for each $p \in \bar{M}$ and each nonzero spacelike vector $X \in$ $\Gamma\left(D_{p}\right)$, the angle $\theta_{p}$ between $J X$ and $D_{p}$ is a constant, that is, independent of the choice of $p \in \bar{M}$ and $X \in \Gamma\left(D_{p}\right)$. In this case, we call the constant angle $\theta_{p}$ the slant angle of the distribution $D_{p}$.

Let $M$ be an immersed submanifold of an almost paraHermitian manifold $\bar{M}$ and $D$ a differentiable distribution on $M$. We denote the orthogonal distribution to $D$ on $M$ by $D^{\perp}$. Then, for all $X \in \Gamma(T M)$, we write

$$
J X=P_{1} f X+P_{2} f X+\omega X,
$$

where $P_{1}$ and $P_{2}$ are orthogonal projections on $D$ and $D^{\perp}$, respectively.

Next, we will give a sufficient and necessary condition for a distribution to be slant.

Theorem 7. Let $M$ be a submanifold of an almost paraHermitian manifold $\bar{M}$ and $D$ a differentiable distribution on 
$M$. Then $D$ is a neutral slant distribution if and only if there exists a constant $\lambda \in[0,1]$ such that

$$
\left(P_{1} f\right)^{2}=\lambda I
$$

Furthermore, in such case, if $\theta$ is the slant angle of $D$ then $\lambda=\cos ^{2} \theta$.

Proof. We suppose that $D$ is a neutral slant distribution on $M$. Then, from (29), we have

$$
\begin{aligned}
\cos \theta(X) & =\frac{g\left(J X, P_{1} f X\right)}{\|J X\|\left\|P_{1} f X\right\|} \\
& =-\frac{g\left(X,\left(P_{1} f\right)^{2} X\right)}{\|J X\|\left\|P_{1} f X\right\|}=\frac{\left\|P_{1} f X\right\|}{\|J X\|},
\end{aligned}
$$

which implies that

$$
\left\|P_{1} f X\right\|=\cos \theta(X)\|J X\|,
$$

for any $X \in \Gamma(D)$. By using (29), (32), and (1), we have

$$
\begin{aligned}
g\left(X,\left(P_{1} f\right)^{2} X\right) & =-g\left(P_{1} f X, P_{1} f X\right) \\
& =-\cos ^{2} \theta(X) g(J X, J X) \\
& =\cos ^{2} \theta(X) g(X, X), \quad \forall X \in \Gamma(D) .
\end{aligned}
$$

Since $g$ is a neutral metric, we obtain

$$
\left(P_{1} f\right)^{2} X=\cos ^{2} \theta(X) X, \quad \forall X \in \Gamma(D) .
$$

If we put $\lambda=\cos ^{2} \theta$, then we have (30).

Conversely, let $\lambda \in[0,1]$ be a constant such that (30) is satisfied. Then, from (1) we have

$$
\begin{aligned}
\cos \theta(X) & =\frac{g\left(J X, P_{1} f X\right)}{\|J X\|\left\|P_{1} f X\right\|} \\
& =-\frac{g\left(X,\left(P_{1} f\right)^{2} X\right)}{\|J X\|\left\|P_{1} f X\right\|}=-\lambda \frac{g(X, X)}{\|J X\|\left\|P_{1} f X\right\|},
\end{aligned}
$$

for any $X \in \Gamma(D)$. Thus we get

$$
\cos \theta(X)=\frac{\lambda\|J X\|}{\left\|P_{1} f X\right\|} .
$$

On the other hand, since $\cos \theta(X)=\left\|P_{1} f X\right\| /\|J X\|$, then we obtain $\cos ^{2} \theta=\lambda$, which implies that $\theta$ is a constant and $D$ is a neutral slant distribution. This completes the proof.

Definition 8. $M$ is called a bineutral slant submanifold of an almost para-Hermitian manifold $\bar{M}$ if there exist two orthogonal distributions $D_{1}$ and $D_{2}$ on $M$ such that

(i) TM admits the orthogonal direct decomposition $T M=D_{1} \oplus D_{2} ;$

(ii) $D_{i}$ is a neutral slant distribution with slant angle $\theta_{i}$ for $i=1,2$.
Given a bineutral slant submanifold $M$, we can write, for any $X \in \Gamma(T M)$,

$$
X=P_{1} X+P_{2} X
$$

where $P_{i}$ denotes the component of $X$ in $D_{i}$ for any $i=1,2$. In particular, if $X \in \Gamma\left(D_{i}\right)$, then we obtain $X_{i}=P_{i} X$. If we define $f_{i}=P_{i} \circ f$, then we have

$$
J X=f_{1} X+f_{2} X+\omega X,
$$

for any $X \in \Gamma(T M)$.

We note that semi-invariant submanifolds are particular cases of bineutral slant submanifolds with slant angles $\theta_{1}=0$ and $\theta_{2}=\pi / 2$.

Theorem 9. Let $M$ be a bineutral slant submanifold with angles $\theta_{1}=\theta_{2}=\theta$. If $g(J X, Y)=0$, for any $X \in \Gamma\left(D_{1}\right)$ and $Y \in \Gamma\left(D_{2}\right)$, then $M$ is slant with angle $\theta$.

Proof. Since $g(J X, Y)=0$, for any $X \in \Gamma\left(D_{1}\right)$ and $Y \in \Gamma\left(D_{2}\right)$, we have $g(f X, Y)=0$; that is, $f X \in \Gamma\left(D_{1}\right)$. Similarly, for $Y \in \Gamma\left(D_{2}\right)$, we find. Then for any $X \in \Gamma(T M), X$ can be written as follows: $X=X_{1}+X_{2}$ such that $X_{1} \in \Gamma\left(D_{1}\right)$ and $X_{2} \in \Gamma\left(D_{2}\right)$ and $\cos ^{2} \theta_{1}=\left\|f X_{1}\right\|^{2} /\left\|J X_{1}\right\|^{2}, \cos ^{2} \theta_{2}=$ $\left\|f X_{2}\right\|^{2} /\left\|J X_{2}\right\|^{2}$. Since $\theta_{1}=\theta_{2}=\theta$, we get

$$
\frac{g(f X, f X)}{g(J X, J X)}=\frac{g\left(f X_{1}, f X_{1}\right)+g\left(f X_{2}, f X_{2}\right)}{g\left(J X_{1}, J X_{1}\right)+g\left(J X_{2}, J X_{2}\right)}=\cos ^{2} \theta,
$$

which gives assertion of the theorem.

Now, as a generalization of semi-invariant submanifolds, we can define semineutral slant submanifolds of an almost para-Hermitian manifold.

Definition 10. $M$ is called a semineutral slant submanifold of an almost para-Hermitian manifold $\bar{M}$ if there exist two orthogonal distributions $D_{1}$ and $D_{2}$ on $M$ such that

(i) $T M$ admits the orthogonal direct sum $T M=D_{1} \oplus D_{2}$,

(ii) the distribution $D_{1}$ is invariant; that is, $J\left(D_{1}\right)=D_{1}$,

(iii) the distribution $D_{2}$ is neutral slant with slant angle $\theta \neq 0$.

In this case, we call $\theta$ the slant angle of submanifold $M$.

It is obvious that the invariant and anti-invariant distributions of a semineutral slant submanifold are neutral slant distributions with the slant angles $\theta=0$ and $\theta=\pi / 2$, respectively.

Now, let $M$ be a semineutral slant submanifold of an almost para-Hermitian manifold $\bar{M}$. Let $M$ be a semislant submanifold with $d_{1} \operatorname{dim}\left(D_{1}\right)$ and $d_{2} \operatorname{dim}\left(D_{2}\right)$. Then we have the following particular cases.

(i) If $d_{2}=0$, then $M$ is an invariant submanifold.

(ii) If $d_{1}=0$ and $\theta=\pi / 2$, then $M$ is an anti-invariant submanifold.

(iii) If $d_{1}=0$ and $\theta \neq \pi / 2$, then $M$ is a proper neutral slant submanifold with slant angle $\theta$. 
(iv) If $d_{1} \cdot d_{2} \neq 0$ and $\theta \neq \pi / 2$, then $M$ is a proper semineutral slant submanifold.

We now give an example of bineutral slant submanifolds.

Example 11. Let $x(u, v, t, s)=(u \sin \alpha, v, u \cos \alpha, 0, s, t \sin \beta$, $0, t \cos \beta)$, where $\alpha$ and $\beta$ are constant. Then, $M$ is a 4 dimensional submanifold of $\bar{M}=R_{4}^{8}$.

By defining

$$
\begin{aligned}
& D_{1}=\left\langle\sin \alpha \frac{\partial}{\partial x_{1}}+\cos \alpha \frac{\partial}{\partial x_{3}}, \frac{\partial}{\partial x_{2}}\right\rangle, \\
& D_{2}=\left\langle\frac{\partial}{\partial x_{5}}, \sin \beta \frac{\partial}{\partial x_{6}}+\cos \beta \frac{\partial}{\partial x_{8}}\right\rangle,
\end{aligned}
$$

we have that $T M=D_{1} \oplus D_{2}$ and $D_{1}, D_{2}$ are neutral slant with slant angles $\cos ^{-1}(|\sin \alpha|)$ and $\cos ^{-1}(|\sin \beta|)$, respectively. Thus $M$ is a bineutral slant submanifold of $\bar{M}$.

Now, let $M$ be a semineutral slant submanifold of an almost para-Hermitian manifold $\bar{M}$ and $P_{i},(i=1,2)$, denoting the orthogonal projections on $D_{i},(i=1,2)$. Then, for any $X \in \Gamma(T M)$, applying $J$ to (37), we have

$$
J X=f P_{1} X+f P_{2} X+\omega P_{2} X
$$

where

$$
J P_{1} X=f P_{1} X, \quad \omega P_{1} X=0 .
$$

From (41) and (42), we have

$$
f X=J P_{1} X+f P_{2} X
$$

By putting $Y=P_{1} Y$ in (20) and $Y=P_{2} Y$ in (21), we get

$$
\begin{array}{ll}
g\left(f X, f P_{1} Y\right)=-\cos ^{2} \theta g\left(X, P_{1} Y\right), & X, Y \in \Gamma(T M), \\
g\left(\omega X, \omega P_{2} Y\right)=-\sin ^{2} \theta g\left(X, P_{2} Y\right), & X, Y \in \Gamma(T M),
\end{array}
$$

respectively.

We give a characterization for the semineutral slant submanifolds of an almost para-Hermitian manifold.

Theorem 12. Let $M$ be an immersed submanifold of an almost para-Hermitian manifold $\bar{M}$. Then $M$ is a semineutral slant submanifold if and only if there exists a constant $\lambda \in[0,1)$ such that $D=\left\{X \in T M \mid f^{2} X=\lambda X\right\}$ is a distribution. Furthermore, in this case, $\lambda=\cos ^{2} \theta$, where $\theta$ denotes slant angle of $M$.

Proof. Let $M$ be a semineutral slant submanifold and $T M=$ $D_{1} \oplus D_{2}$, where $D_{1}$ is invariant and $D_{2}$ is neutral slant. We put $\lambda=\cos ^{2} \theta$, where $\theta$ denotes slant angle of $M$. For any $X \in \Gamma(D)$, if $X \in \Gamma\left(D_{1}\right)$, then we have

$$
X=J^{2} X=f^{2} X=\lambda X,
$$

which implies that $\lambda=1$. But this is a contradiction that $\lambda \epsilon$ $[0,1)$. Therefore we obtain $D \subseteq D_{2}$. On the other hand, since $D_{2}$ is a neutral slant distribution, it follows from Theorem 7 that $f^{2} X=\left(f P_{2}\right)^{2} X=\lambda X$, which means that $D_{2} \subseteq D$. Thus $D=D_{2}$ is a distribution.

Conversely, we can consider the orthogonal direct decomposition $T M=D \oplus D^{\perp}$. It is obvious that $f D \subseteq D$, from which we have $g(J X, Y)=-g(X, J Y)=-g(X, f Y)=0$ for any $X \in \Gamma\left(D^{\perp}\right)$ and $Y \in \Gamma(D)$. Hence $D^{\perp}$ is an invariant distribution. Finally, Theorem 7 imply that $D$ is a neutral slant distribution, with slant angle $\theta$ satisfying $\lambda=\cos ^{2} \theta$. ation.

We can easily present some examples of the above situ-

Example 13. $x(u, v, t, r)=(u, 0, u, v \sin \theta, 0, v \cos \theta, t, s)$, $\theta \neq \pi / 2$ defines a four-dimensional proper semineutral slant submanifold $M$, with slant angle $\cos ^{-1}(|\sin \theta / \sqrt{2}|)$, in $R_{4}^{8}$.

Moreover, it is easy to see that

$$
\begin{gathered}
X_{1}=\frac{\partial}{\partial x_{7}}, \quad X_{3}=\frac{\partial}{\partial x_{1}}+\frac{\partial}{\partial x_{3}}, \\
X_{2}=\frac{\partial}{\partial x_{8}}, \quad X_{4}=\sin \theta \frac{\partial}{\partial x_{4}}+\cos \theta \frac{\partial}{\partial x_{6}}
\end{gathered}
$$

from a local orthogonal frame of TM. Then, we can define $D_{1}=\operatorname{Span}\left\{X_{1}, X_{2}\right\}$ and $D_{2}=\operatorname{Span}\left\{X_{3}, X_{4}\right\}$.

Example 14. $x(u, v, t, s)=(u, v, t \sin \alpha, s \cos \beta, t \cos \alpha$, $s \sin \beta, 0,0)$ defines a four-dimensional proper semineutral slant submanifold $M$, with slant angle $\cos \theta=|\sin (\alpha+\beta)|$, in $R_{4}^{8}$, where $\alpha$ and $\beta$ are constant.

Moreover it is easy to see that

$$
\begin{array}{ll}
X_{1}=\frac{\partial}{\partial x_{1}}, & X_{3}=\sin \alpha \frac{\partial}{\partial x_{3}}+\cos \alpha \frac{\partial}{\partial x_{5}} \\
X_{2}=\frac{\partial}{\partial x_{2}}, & X_{4}=\cos \beta \frac{\partial}{\partial x_{4}}+\sin \beta \frac{\partial}{\partial x_{6}}
\end{array}
$$

from a local orthogonal frame of TM. Then we can define $D_{1}=\operatorname{Span}\left\{X_{1}, X_{2}\right\}$ and $D_{2}=\operatorname{Span}\left\{X_{3}, X_{4}\right\}$.

Then, it is easy to show that all conditions of Theorem 12 are satisfied.

Next, we will give useful characterizations for integrable conditions of distributions.

Theorem 15. Let $M$ be a semineutral slant submanifold of a para-Kähler manifold $\bar{M}$. Then we have the following:

(a) the distribution $D_{1}$ is integrable if and only if

$$
h(X, f Y)=h(f X, Y) \text {, }
$$

for any $X, Y \in \Gamma\left(D_{1}\right)$,

(b) the distribution $\mathrm{D}_{2}$ is integrable if and only if

$$
P_{1}\left(\nabla_{X} f P_{2} Y-\nabla_{Y} f P_{2} X\right)=P_{1}\left(A_{\omega P_{2} Y} X-A_{\omega P_{2} X} Y\right) \text {, }
$$

for any $X, Y \in \Gamma\left(D_{2}\right)$. 
Proof. From (2), we get

$$
\bar{\nabla}_{X} J Y=J \bar{\nabla}_{X} Y
$$

for all $X, Y \in \Gamma(T \bar{M})$.

(a) By using Gauss-Weingarten formulas, (5), and (6) in (50), we have

$$
\begin{aligned}
\nabla_{X} f Y & +h(X, f Y) \\
& =f \nabla_{X} Y+\omega \nabla_{X} Y+B h(X, Y)+C h(X, Y),
\end{aligned}
$$

for any $X, Y \in \Gamma\left(D_{1}\right)$. From (41) and (51), we obtain

$$
\begin{aligned}
\nabla_{X} f Y+h(X, f Y)= & f P_{1} \nabla_{X} Y+f P_{2} \nabla_{X} Y+\omega P_{2} \nabla_{X} Y \\
& +B h(X, Y)+C h(X, Y)
\end{aligned}
$$

By equating the normal part of the last equation, we have

$$
h(X, f Y)=\omega P_{2} \nabla_{X} Y+C h(X, Y) .
$$

If we change the role of $X$ and $Y$ in (53), we write

$$
h(f X, Y)=\omega P_{2} \nabla_{Y} X+C h(Y, X) .
$$

Since $h$ is symmetric, from (53) and (54), we get

$$
h(X, f Y)-h(f X, Y)=\omega P_{2}[X, Y], \quad \forall X, Y \in \Gamma\left(D_{1}\right) .
$$

Assume that the distribution $D_{1}$ is integrable. Then, for any $X, Y \in \Gamma\left(D_{1}\right)$, we have $[X, Y] \in \Gamma\left(D_{1}\right)$ which implies that $\omega P_{2}[X, Y]=0$. Thus from (55) we obtain (48).

Conversely, if (48) is satisfied, then from (55), we have $\omega P_{2}[X, Y]=0$, for any $X, Y \in \Gamma\left(D_{1}\right)$, which implies that $P_{2}[X, Y]=0$. Then we conclude that $[X, Y] \in \Gamma\left(D_{1}\right)$.

(b) From (41) and Gauss-Weingarten formulae, we have

$$
\begin{aligned}
\bar{\nabla}_{X} J Y= & \nabla_{X} J P_{1} Y+h\left(X, J P_{1} Y\right)+\nabla_{X} f P_{2} Y+h\left(X, f P_{2} Y\right) \\
& -A_{\omega P_{2} Y} X+\nabla_{X}^{\perp} \omega P_{2} Y
\end{aligned}
$$

for all $X, Y \in \Gamma(T M)$. On the other hand, by using (5) and (6), we write

$$
J \bar{\nabla}_{X} Y=f \nabla_{X} Y+\omega \nabla_{X} Y+B h(X, Y)+C h(X, Y)
$$

By using (56) and (57) in (50), we get

$$
\begin{aligned}
\nabla_{X} f P_{2} Y+h\left(X, f P_{2} Y\right)-A_{\omega P_{2} Y} X+\nabla_{X}^{\perp} \omega P_{2} Y \\
=f \nabla_{X} Y+\omega \nabla_{X} Y+B h(X, Y)+C h(X, Y),
\end{aligned}
$$

for any $X, Y \in \Gamma\left(D_{2}\right)$. Since $h$ is symmetric we obtain

$$
f[X, Y]=\nabla_{X} f P_{2} Y-\nabla_{Y} f P_{2} X+A_{\omega P_{2} X} Y-A_{\omega P_{2} Y} X
$$

which gives

$$
\begin{aligned}
P_{1} f[X, Y]= & P_{1}\left\{\nabla_{X} f P_{2} Y-\nabla_{Y} f P_{2} X\right\} \\
& -P_{1}\left\{A_{\omega P_{2} Y} X-A_{\omega P_{2} X} Y\right\}
\end{aligned}
$$

Let the distribution $D_{2}$ be integrable. Then $P_{1} f[X, Y]=0$, for all $X, Y \in \Gamma\left(D_{2}\right)$, and hence from (60), the equation (49) is obvious.

Conversely, if (49) is satisfied then $P_{1} f[X, Y]=0$; that is, $[X, Y] \in \Gamma\left(D_{2}\right)$ for any $X, Y \in \Gamma\left(D_{2}\right)$. This completes the proof.

Definition 16. Let $M$ be a semi-invariant submanifold of an almost para-Hermitian manifold $\bar{M}$. Then we say that

(i) $M$ is $D_{1}$-geodesic if

$$
h(X, Y)=0, \quad \forall X, Y \in \Gamma\left(D_{1}\right),
$$

(ii) $M$ is $D_{2}$-geodesic if

$$
h(X, Y)=0, \quad \forall X, Y \in \Gamma\left(D_{2}\right),
$$

(iii) $M$ is mixed geodesic if

$$
h(X, Y)=0, \quad \forall X \in \Gamma\left(D_{1}\right), Y \in \Gamma\left(D_{2}\right) .
$$

Lemma 17. Let $M$ be a mixed-geodesic semineutral slant submanifold of a para-Kähler manifold $\bar{M}$. Then the distribution $D_{1}$ is integrable if and only if

$$
J A_{N} X=-A_{N} J X
$$

for any $X \in \Gamma\left(D_{1}\right)$ and $N \in \Gamma\left(T^{\perp} M\right)$.

Proof. Since $M$ is a mixed-geodesic submanifold, from (4) we find that $A_{N} X$ has no component on $D_{2}$. By using (4) and (1), we obtain

$$
\begin{gathered}
g\left(J A_{N} X, Y\right)=-g\left(A_{N} X, J Y\right)=-g(h(X, J Y), N), \\
g\left(A_{N} J X, Y\right)=g(h(J X, Y), N) .
\end{gathered}
$$

Thus, we can write

$$
g\left(J A_{N} X+A_{N} J X, Y\right)=g(h(J X, Y)-h(X, J Y), N),
$$

for all $X, Y \in \Gamma\left(D_{1}\right)$. Taking into account Theorem 15(a) and the last equation, the proof is completed.

Theorem 18. Let $M$ be a semineutral slant submanifold of a para-Kähler manifold $\bar{M}$. If $\nabla \omega=0$, then $M$ is a mixedgeodesic submanifold. Furthermore,

(a) if $X, Y \in \Gamma\left(D_{1}\right)$, then either $M$ is a $D_{1}$-geodesic submanifold or $h(X, Y)$ is an eigenvector of $C^{2}$ with the eigenvalue 1 ,

(b) if $X, Y \in \Gamma\left(D_{2}\right)$, then either $M$ is a $D_{2}$-geodesic submanifold or $h(X, Y)$ is an eigenvector of $C^{2}$ with the eigenvalue $\cos ^{2} \theta$. 
Proof. If $\nabla \omega=0$, then from (13) we get $C h(X, Y)=h(X, f Y)$, for all $X, Y \in \Gamma(T M)$. Since $D_{1}$ is an invariant and $D_{2}$ is a neutral slant distribution with the slant angle $\theta$, we obtain

$$
\begin{aligned}
C^{2} h(X, Y) & =C h(X, f Y)=h\left(X, f^{2} Y\right) \\
& =h\left(X, \cos ^{2} \theta Y\right)=\cos ^{2} \theta h(X, Y), \\
C^{2} h(X, Y) & =C^{2} h(Y, X)=C h(Y, f X) \\
& =h\left(Y, f^{2} X\right)=h(Y, X)=h(X, Y),
\end{aligned}
$$

for any $X \in \Gamma\left(D_{1}\right), Y \in \Gamma\left(D_{2}\right)$. By using (67) we get

$$
\sin ^{2} \theta h(X, Y)=0
$$

which implies that $h(X, Y)=0$, for any $X \in \Gamma\left(D_{1}\right), Y \in$ $\Gamma\left(D_{2}\right)$, that is, $M$ is mixed-geodesic. Similarly, we obtain

$$
C^{2} h(X, Y)=h(X, Y),
$$

for all $X, Y \in \Gamma\left(D_{1}\right)$, and

$$
C^{2} h(X, Y)=\cos ^{2} \theta h(X, Y),
$$

for all $X, Y \in \Gamma\left(D_{2}\right)$. This completes the proof.

Proposition 19. Let $M$ be a semineutral slant submanifold of a para-Kähler manifold $\bar{M}$. Then $\nabla \omega=0$ if and only if

$$
A_{\mathrm{CN}} Z=-A_{N} f Z,
$$

for all $Z \in \Gamma(T M), N \in \Gamma\left(T^{\perp} M\right)$.

Proof. From (13) and (1),we get

$$
\begin{aligned}
g\left(\left(\nabla_{X} \omega\right) Z, N\right) & =g(C h(X, Z)-h(X, f Z), N) \\
& =-g(h(X, Z), C N)-g(h(X, f Z), N),
\end{aligned}
$$

for any $X, Z \in \Gamma(T M), N \in \Gamma\left(T^{\perp} M\right)$. Taking into account (4), we get

$$
g\left(\left(\nabla_{X} \omega\right) Z, N\right)=-g\left(A_{C N} Z+A_{N} f Z, X\right),
$$

which completes the proof.

Proposition 20. Let $M$ be a semineutral slant submanifold of a para-Kähler manifold $\bar{M}$. Then $\nabla f=0$ if and only if

$$
A_{\omega P_{2} Y} Z=A_{\omega P_{2} Z} Y
$$

for all $Y, Z \in \Gamma(T M)$.

Proof. From (12) and (1) we have

$$
\begin{aligned}
g\left(\left(\nabla_{X} f\right) Y, Z\right) & =g\left(A_{\omega Y} X+B h(X, Y), Z\right) \\
& =g\left(A_{\omega P_{2} Y} X, Z\right)-g\left(h(X, Y), \omega P_{2} Z\right) \\
& =g\left(A_{\omega P_{2} Y} X, Z\right)-g\left(A_{\omega P_{2} Z} X, Y\right),
\end{aligned}
$$

for any $X, Y, Z \in \Gamma(T M)$. Since the $A$ is symmetric then we obtain from the last equation

$$
g\left(\left(\nabla_{X} f\right) Y, Z\right)=g\left(A_{\omega P_{2} Y} Z-A_{\omega P_{2} Z} Y, X\right) .
$$

This completes the proof.

Proposition 21. Let $M$ be a semineutral slant submanifold of a para-Kähler manifold $\bar{M}$. If $\nabla f=0$ then the distributions are integrable and their leaves are totally geodesic in $M$.

Proof. Since $\nabla f=0$, then from (12) we obtain $B h(X, Y)=0$ for any $X \in \Gamma(T M)$ and $Y \in \Gamma\left(D_{1}\right)$. By using (1) and (5), we have

$$
0=(B h(X, Y), Z)=g(J h(X, Y), Z)=-g(h(X, Y), J Z),
$$

where $X, Z \in \Gamma(T M)$ and $Y \in \Gamma\left(D_{1}\right)$. Thus one can easily see that

$$
\begin{gathered}
g\left(h(X, Y), \omega P_{2} Z\right)=0, \\
g\left(J h(X, Y), \omega P_{2} Z\right)=0 .
\end{gathered}
$$

Since $\bar{M}$ is a para-Kähler manifold, taking into account (78), we get

$$
\begin{aligned}
& 0=g\left(J h(X, Y), \omega P_{2} \nabla_{X} Y\right) \\
& 0=-g\left(\omega P_{2} \nabla_{X} Y, \omega P_{2} \nabla_{X} Y\right) \\
& 0=\sin ^{2} \theta g\left(P_{2} \nabla_{X} Y, P_{2} \nabla_{X} Y\right),
\end{aligned}
$$

which gives $P_{2} \nabla_{X} Y=0$; that is, $\nabla_{X} Y \in \Gamma\left(D_{1}\right)$. Now, let $Y \in \Gamma\left(D_{1}\right)$ and $V \in \Gamma\left(D_{2}\right)$. Since $D_{1}$ is orthogonal to $D_{2}$, the induced metric on $M$ is the neutral metric, and it is easy to see that $\nabla_{Z} V \in \Gamma\left(D_{2}\right)$. Hence the proof is complete.

\section{Acknowledgment}

The author is grateful to the referees for their valuable comments and suggestions.

\section{References}

[1] B.-Y. Chen, "Slant immersions," Bulletin of the Australian Mathematical Society, vol. 41, no. 1, pp. 135-147, 1990.

[2] B.-Y. Chen, Geometry of Slant Submanifolds, Katholieke Universiteit Leuven, Louvain, Belgium, 1990.

[3] M. Atçeken, "Semi-slant submanifolds of an almost paracontact metric manifold," Canadian Mathematical Bulletin, vol. 53, no. 2, pp. 206-217, 2010.

[4] H. Li and X. Liu, "Semineutral Slant Submanifolds of Almost Para-Hermitian Manifolds," Georgian Mathematical Journal, vol. 12, no. 2, pp. 273-282, 2005.

[5] B. Şahin and S. Keleş, "Slant submanifolds of Kaehler product manifolds," Turkish Journal of Mathematics, vol. 31, no. 1, pp. 6577, 2007.

[6] N. Papaghiuc, "Semi-slant submanifolds of a Kaehlerian manifold," Analele Ştiinţifice ale Universităţii Al. I. Cuza din Iaşi. Serie Nouă. Matematică, vol. 40, no. 1, pp. 55-61, 1994. 
[7] A. Lotta, "Slant submanifolds in contact geometry," Bulletin Mathématique de la Société des Sciences Mathématiques de Roumanie, vol. 39, pp. 183-198, 1996.

[8] A. Lotta, "Three-dimensional slant submanifolds of $K$-contact manifolds," Balkan Journal of Geometry and its Applications, vol. 3, no. 1, pp. 37-51, 1998.

[9] J. L. Cabrerizo, A. Carriazo, L. M. Fernández, and M. Fernández, "Slant submanifolds in Sasakian manifolds," Glasgow Mathematical Journal, vol. 42, no. 1, pp. 125-138, 2000.

[10] J. L. Cabrerizo, A. Carriazo, L. M. Fernández, and M. Fernández, "Semi-slant submanifolds of a Sasakian manifold," Geometriae Dedicata, vol. 78, no. 2, pp. 183-199, 1999.

[11] K. Arslan, A. Carriazo, B.-Y. Chen, and C. Murathan, "On slant submanifolds of neutral Kaehler manifolds," Taiwanese Journal of Mathematics, vol. 14, no. 2, pp. 561-584, 2010.

[12] V. Cruceanu, P. Fortuny, and P. M. Gadea, "A survey on paracomplex geometry," The Rocky Mountain Journal of Mathematics, vol. 26, no. 1, pp. 83-115, 1996.

[13] S. Ivanov and S. Zamkovoy, "Parahermitian and paraquaternionic manifolds," Differential Geometry and Its Applications, vol. 23, no. 2, pp. 205-234, 2005. 


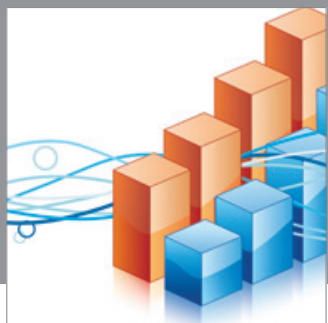

Advances in

Operations Research

mansans

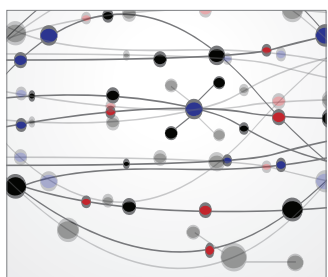

The Scientific World Journal
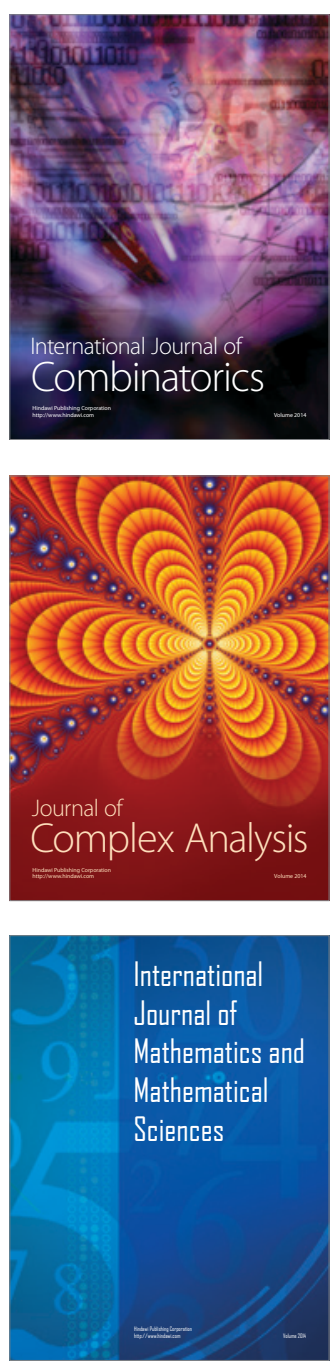
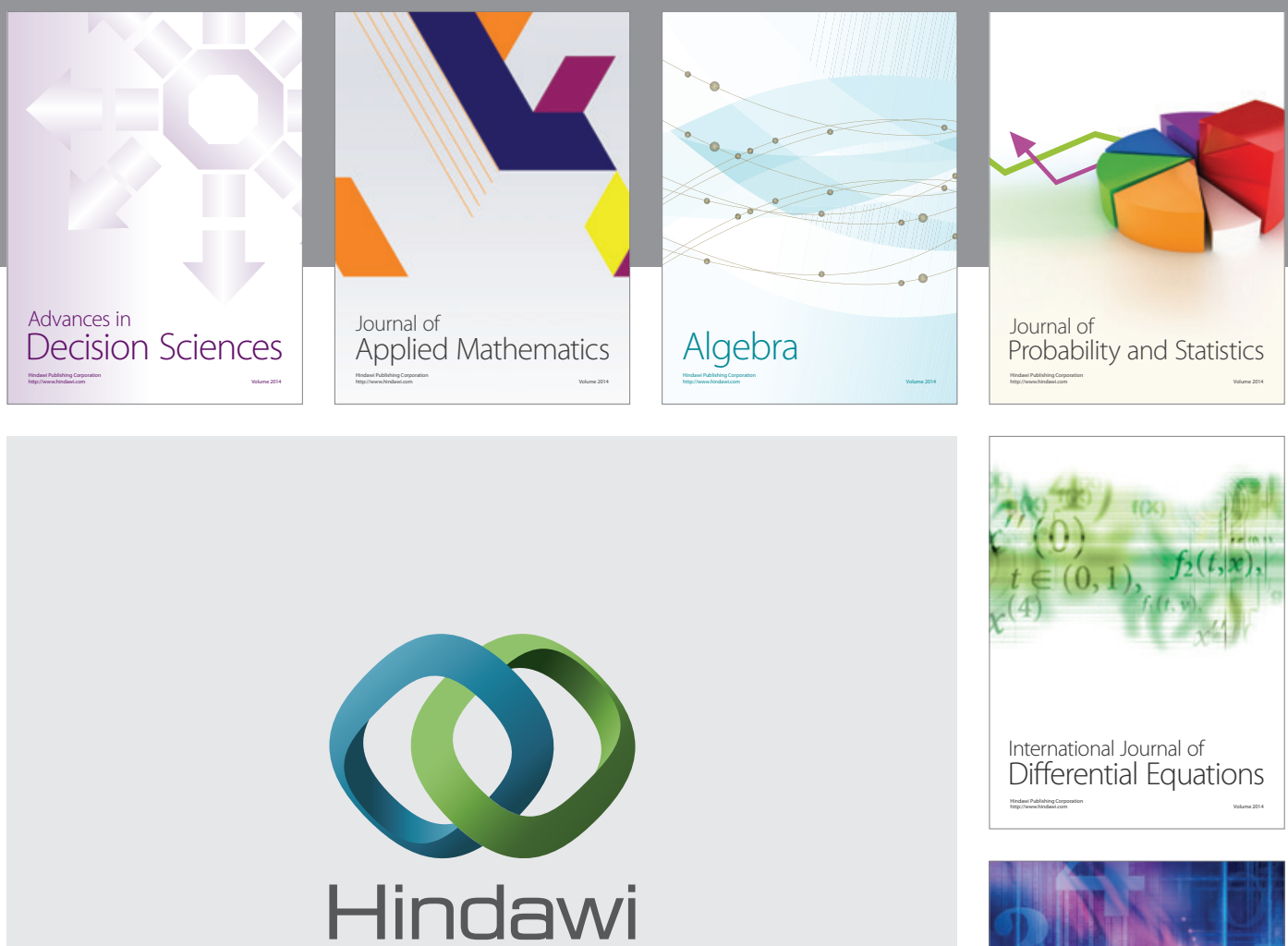

Submit your manuscripts at http://www.hindawi.com
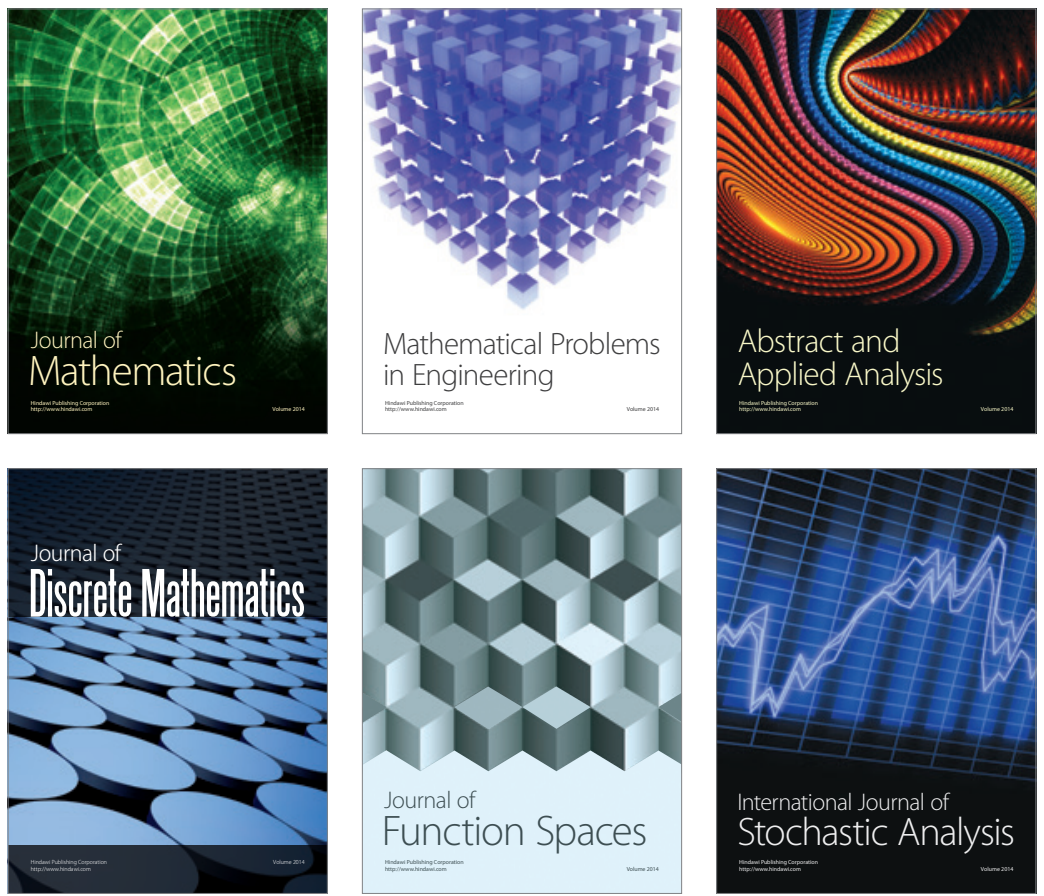

Journal of

Function Spaces

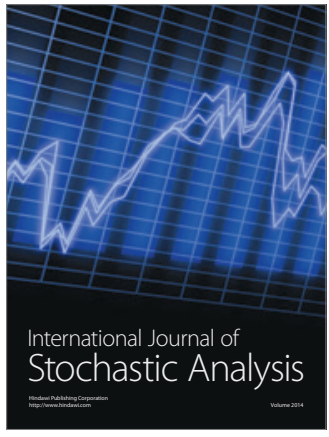

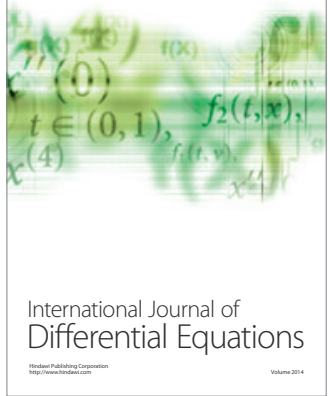
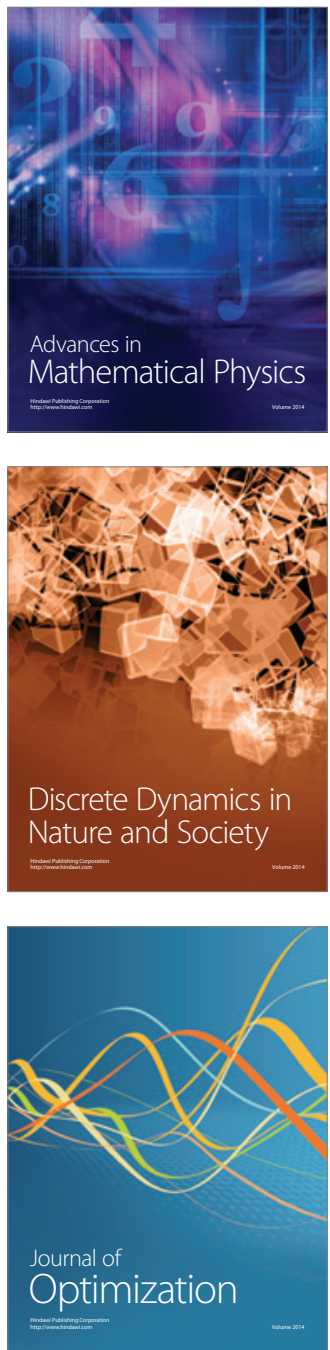